\title{
PERCEPÇÃO DA POPULAÇÃO EM GERAL E DOS PROFISSIONAIS DE SAÚDE SOBRE A FORMA DE OBTENÇÃO DE ÓRGÃOS PARA TRANSPLANTE: A PERSPECTIVA MERCADOLÓGICA
}

\author{
Perception of the general population and health professionals on how to obtain \\ organs for transplantation: a marketing perspective
}

Daniela Alves Pereira de Andrade ${ }^{1}$, José Roberto Goldim²

\section{RESUMO}

Objetivo: Apresentar as percepções de profissionais de saúde e da população em geral com relação à forma de obtenção de órgãos, em especial a abordagem de mercado. Métodos: Buscamos apresentar os dados relativos à percepção dos participantes de forma desinteressada e com a inclusão do envolvimento de necessidade pessoal ou familiar para obtenção de órgãos para fins de transplante, utilizando a abordagem de mercado. Para atender aos objetivos deste estudo, foi elaborado um questionário para coletar a opinião dos participantes. Sua distribuição foi realizada pessoalmente de forma aleatória, e também foi elaborada uma versão eletrônica divulgada via página no Facebook. A análise das respostas obtidas foram discutidas ao nível de $5 \%$ de significância e consideradas significativas quando o valor de $\mathrm{p}$ foi <0,05. Resultados: Ao todo, 692 pessoas participaram da pesquisa. Na categoria do profissional de saúde há maior tendência do que na população em geral em discordar dos incentivos indiretos relacionados à redução de impostos e licença remunerada de 30 dias. Estas foram as únicas associações significativas referentes aos profissionais da saúde. A maioria dos participantes $(80,1 \%)$ concordou que a doação de órgãos deve ser um ato desinteressado e estritamente solidário, mas $52 \%$ acredita que o mercado poderia ser um sistema justo e benéfico para todos. Em uma situação extrema de carência absoluta de órgãos, 54,9\% dos participantes indicaram que pagariam por um órgão para salvar sua vida ou a vida de algum familiar. Conclusão: Coletivamente, os participantes apoiam amplamente a doação solidária, condenam a possibilidade de pagamento ou remuneração associada à captação, mas a maioria das pessoas pagaria para obter um órgão em caso de extrema necessidade pessoal ou de seus familiares. Isto demonstra a necessidade de um aprofundamento da reflexão sobre os aspectos éticos associados às questões envolvidas na captação de órgãos para fins de transplantes.

Descritores: Doação de Órgãos; Doação de Tecidos; Transplante.

\footnotetext{
Instituição:

${ }^{1}$ Laboratório de Pesquisa em Bioética, Hospital de Clínicas de Porto Alegre/RS/Brasil

2 Programa de Pós-Graduação em Medicina: Ciências Médicas, Universidade Federal do Rio Grande do Sul/Brasil, Laboratório de Pesquisa em Bioética, Hospital de Clínicas de Porto Alegre/RS/ Brasil.
}

\section{Correspondência:}

Daniela Alves Pereira de Andrade

Laboratório de Bioética e Ética na Ciência, Centro de Pesquisas, Hospital de Clínicas de Porto Alegre

Rua Ramiro Barcelos, 2350 - 90035-903 Porto Alegre - RS - Brasil Tel.:: (51) 33598543

E-mail: alves@ceiri.com.br

Recebido em: 30/01/2018

Aceito em: 01/03/2018

\section{INTRODUÇÃO}

Considerado um dos milagres da Medicina do século XX, o transplante prolongou e melhorou a vida de milhões de pacientes em todo o mundo, sendo considerado o melhor tratamento e, em muitos casos, o único possível para o problema de muitos órgãos vitais quando se encontram em condições irreversíveis, colocando um paciente em estado terminal. ${ }^{1}$

As possibilidades geradas pelo desenvolvimento tecnológico, pelas inovações científicas, pela ampla disseminação de novos equipamentos, de drogas e procedimentos cirúrgicos que objetivam prolongar a vida humana acabaram acompanhadas da escassez de doadores. ${ }^{2,3}$ 
Percepção da população em geral e dos profissionais de saúde sobre a forma de obtenção de órgãos para transplante: a perspectiva mercadológica

Atualmente, os transplantes de órgãos geram uma série de questionamentos éticos acerca da origem dos órgãos, da forma de obtenção do material a ser transplantado e do tipo de procedimento a ser realizado. ${ }^{4}$

Com relação à origem de que "os órgãos podem ser oriundos de outras espécies animais (xenotransplante), de seres humanos vivos (alotransplante intervivos) ou mortos (alotransplante de doador cadáver)" e no que se refere à forma de obtenção, especificamente falando em órgãos oriundos de seres humanos, enfatiza-se que "a questão mais importante é a do resguardo da voluntariedade e da espontaneidade no ato de doar órgãos, ou a aceitação que o bem comum está acima da vontade do indivíduo e permitir a apropriação dos órgãos de cadáveres ou que o indivíduo é proprietário do seu corpo e, dessa forma, pode dispor do mesmo como melhor lhe aprouver".5,6

O tempo de espera para obtenção de órgão para transplante constitui-se um dos grandes problemas relacionados à área da Saúde e uma constante em diversos países, inclusive no Brasil. O número de pessoas que aguardam um órgão e até mesmo falecem à espera de um transplante aumenta consideravelmente todos os anos, enquanto o número de doadores voluntários não cresce na mesma medida da necessidade de órgãos. Diante desse quadro, houve o surgimento de um mercado ilícito de venda de órgãos, em que os compradores e intermediadores dirigirem-se aos países onde existe uma porcentagem grande de população de baixa renda e péssimas condições de vida com o objetivo de adquirir órgãos de indivíduos vivos mediante pagamento. ${ }^{7}$

No Brasil, a abordagem de mercado vem sendo estudada

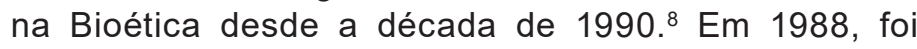
aprovada uma lei estadual no Rio Grande do Sul/Brasil, que previa o pagamento das despesas de funeral às famílias que doassem órgãos. ${ }^{9}$ Diferentes abordagens vêm sendo estabelecidas, desde a compra direta, como na Índia, até o controle estatal do mercado utilizado no Irã. ${ }^{10,11}$ Outras abordagens também estão presentes, como a de oferecer benefícios indiretos, utilizada em diversas legislações dos Estados Unidos. ${ }^{12}$

Esse panorama ainda continua controverso, com posições que defendem a regulamentação do mercado de órgãos ${ }^{13-17}$ e outras que são contra tal procedimento. ${ }^{18-22}$ Contudo, é possível identificar alguns pontos em comum, especialmente, sobre a necessidade de estudos-piloto para demonstrar se seria possível ou não a organização de um mercado de órgãos eticamente adequado. ${ }^{13,23-26}$

A questão da regulamentação do mercado de órgãos apresenta-se como um intenso debate da atualidade. Dessa forma, buscamos neste artigo apresentar as percepções de profissionais de saúde e da população em geral com relação à forma de obtenção de órgãos para transplante, em especial a abordagem de mercado, com a finalidade de instrumentalizar esse debate.

\section{MÉTODOS}

Foi elaborado um questionário estruturado com 10 perguntas, sendo três para definir as características do participante (idade, sexo e se é profissional da Saúde ou não) e as sete restantes com temas sobre captação de órgãos. Foi utilizada uma escala de Likert de três pontos: concordo, discordo e não tenho opinião.

As questões utilizadas foram as seguintes:

"Você considera que a doação de órgãos deve ser um ato desinteressado e estritamente solidário?", denominada de variável "solidariedade";

"Você considera que o mercado de órgãos poderia ser sistema justo e benéfico para todos, visando ampliar a possibilidade de realização de transplantes?", denominada de variável "mercado". "Na Índia é possível comprar diretamente um órgão de uma pessoa para realização de transplantes intervivos. Qual a sua opinião?”, denominada de variável "Índia".

"No Irã o governo é quem compra e regula o mercado de órgãos para transplantes. Qual a sua opinião?", denominada de variável "Irã".

"Em vários estados norte-americanos existem benefícios de redução de impostos da ordem de US\$10.000,00 (dez mil dólares), para quem disponibiliza um órgão para realização de transplantes intervivos. Qual a sua opinião?”, denominada de variável "imposto".

"Em vários estados norte-americanos um funcionário público, que doa órgão para fins de transplante intervivos, ganha uma licença remunerada de 30 dias, percebendo o valor integral de seu salário. Qual a sua opinião?", denominada de variável "licença".

"Em uma situação extrema, de carência absoluta de órgãos para fins de transplante, você pagaria por um órgão para salvar a sua via ou a vida de algum familiar?", denominada de variável "necessidade".

Os dados foram obtidos de forma aleatória por meio de abordagem direta ou utilizando um formulário eletrônico criado no sistema Survey Monkey, disseminado por uma página no Facebook com o título "Coleta de Opiniões sobre Transplantes de Órgãos" (https://www. facebook.com/pages/Coleta-de-Opini\%C3\%B5essobre-Transplantes-de-\%C3\%93rg\%C3\%A3os/32 
9606923876148?ref=hl).27 Essa página foi criada exclusivamente para a divulgação do questionário, captando de forma aleatória pessoas com interesse em participar da pesquisa. Não houve qualquer tipo de intervenção que pudesse influenciar nas respostas do participante.

O estudo das respostas dos participantes nas diferentes questões objetivas apresentadas foi realizado estabelecendo a distribuição de frequências dos dados e verificadas associações pelo teste do Qui quadrado utilizando o programa de análise estatística SPSS. O nível de significância utilizado foi de 5\% $(P<0,05)$.

\section{RESULTADOS}

O questionário foi respondido anonimamente por 692 pessoas entre setembro e outubro de 2014. Dessas 692 respostas, 292 foram obtidas com os formulários físicos distribuídos pessoalmente e as demais 400 respostas foram obtidas pelo formulário eletrônico. Não foram verificadas associações estatisticamente significativas que sejam decorrentes do tipo de coleta utilizada $(P>0,05)$.

Dos 692 participantes, 470 (67,9\%) foram do sexo feminino e $222(32,1 \%)$ do masculino. A faixa etária variou de 17 a 79 anos, com uma média de 37,42 $\pm 13,9$ anos. O número de pessoas que se identificaram como profissional da saúde foi de 345 (49,9\%).

A maioria dos participantes, $554(80,1 \%)$, indicou que concorda que a doação de órgãos deve ser um ato desinteressado e estritamente solidário. Por outro lado, 360 participantes $(52,0 \%)$ acreditam que o mercado de órgãos poderia ser um sistema justo e benéfico para todos, visando ampliar a possibilidade de realização de transplantes.

Ao abordarmos exemplos de mercado de órgãos no mundo, 540 participantes $(78,0 \%)$ discordam da possibilidade de compra do órgão de uma pessoa para realização de transplantes intervivos. Continuando na mesma perspectiva mercadológica, 493 pessoas $(71,2 \%)$ discordaram da iniciativa do governo iraniano que compra e regula o mercado de órgãos para transplantes.

Saindo da perspectiva mercadológica, ou seja, do pagamento pelo órgão, e ingressando nas práticas que promovem formas de compensação pelo ato da doação, 323 participantes $(46,7 \%)$ discordam da iniciativa de vários estados norte-americanos onde existem benefícios de redução de impostos da ordem de US $\$ 10.000,00$ (dez mil dólares) para quem disponibiliza um órgão para realização de transplantes intervivos.
Continuando com a perspectiva da compensação, 356 participantes $(51,4 \%)$ concordam com a iniciativa de vários estados norte-americanos, onde um funcionário público que doa órgão para fins de transplante intervivos ganha uma licença remunerada de 30 dias, recebendo o valor integral de seu salário. Essa é a primeira variável dentre os exemplos reais apresentados, em que a resposta "concorda" é a mais frequente.

Ao sair do campo das possibilidades e entrando no campo de uma necessidade individual concreta, 380 participantes $(54,9 \%)$ indicaram que em uma situação extrema de carência absoluta de órgãos para fins de transplante, pagariam por um órgão para salvar sua vida ou a vida de algum familiar. As demais respostas foram assinaladas por $167(24,1 \%)$ participantes que discordaram e 140 (20,2\%) que indicaram não possuir opinião sobre o assunto. Vale destacar que esse foi o maior índice de respostas indicando não possuir opinião em todas as questões apresentadas.

Cabe destacar que as respostas dadas pelos profissionais de saúde tiveram associações significativas apenas com duas variáveis: benefícios de redução de impostos para o doador ( $\mathrm{X} 2=7,558 ; \mathrm{P}<0,05)$ e licença remunerada de 30 dias $(X 2=6,016 ; P<0,05)$. Todas as demais avaliações sobre eventuais associações entre as demais variáveis foram não-significativas $(P>0.05)$ programadas.

\section{DISCUSSÃO}

Comparando os dados obtidos, destaca-se a alta aceitação para a doação solidária e a alta discordância na possibilidade de compra de órgãos na Índia e Irã (Figura 1).

Figura 1: Distribuição comparativa das respostas às diferentes situações apresentadas sobre captação de órgãos

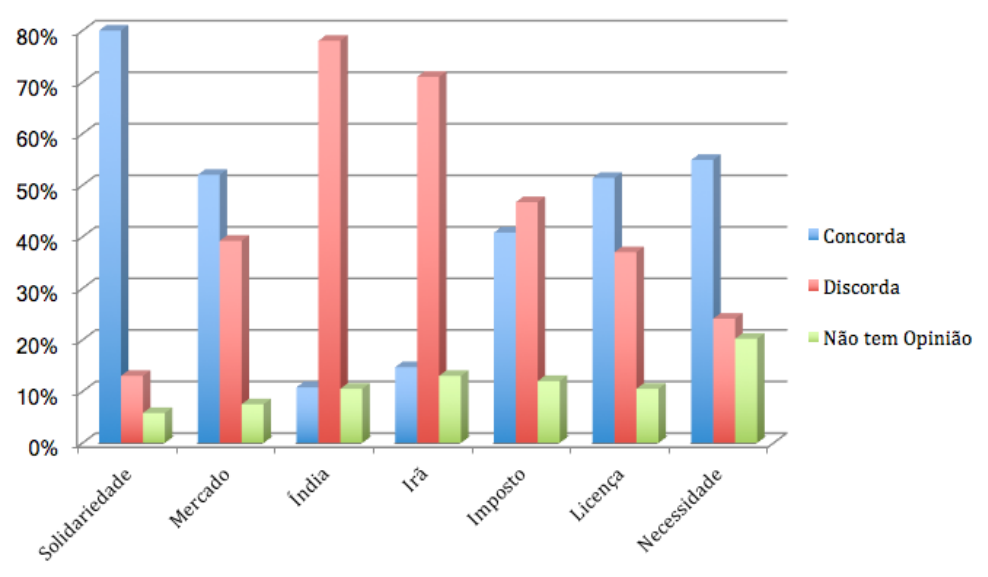

$(n=692)$ 
A alta aceitação à doação solidária é seguida por uma queda de aceitação referente ao mercado de órgãos e uma tendência ainda menor nos casos da Índia e do Irã, ainda que nesse último tenha ocorrido um leve aumento da aceitação em relação à Índia. Nas possibilidades que utilizam benefícios indiretos, como a redução de impostos e licença remunerada, é possível observar uma tendência de aceitação maior do que as que utilizam remuneração diretas, como nos casos da Índia e do Irã. Em 2012, 63,6\% de uma amostra de norte-americanos declarou que os incentivos financeiros não afetariam a sua decisão de doar seus órgãos. ${ }^{28}$

$\mathrm{Na}$ perspectiva coletiva, os participantes indicaram alta aceitação à doação solidária, mas na perspectiva individual, com um fato concreto de necessidade, há aceitação significativa $(54,9 \%)$ ao pagamento para a obtenção de órgão. A aceitação nesse último caso não é mais significativa que a da doação solidária, mas indica que uma parcela significativa dos participantes tenderia a adotar a alternativa do pagamento em um caso extremo. Por outro lado, é interessante notar que a questão da existência de uma necessidade individual, com o pagamento para obtenção de órgãos, não ultrapassou a perspectiva coletiva da doação solidária.

Do ponto de vista bioético, essas respostas podem merecer múltiplos enquadramentos teóricos, ressaltando a necessidade de uma abordagem complexa para a avaliação desses aspectos.

A resposta à questão da solidariedade pode ser justificada desde a perspectiva do Intuicionismo ou da Ética da Situação. ${ }^{29}$ A solidariedade também pode ser justificada como uma Virtude, especialmente por meio da generosidade e compaixão; ${ }^{30}$ como o Princípio da Beneficência - fazer o bem; ${ }^{31}$ como um Direito Humano transpessoal; ${ }^{32}$ como Ética da Responsabilidade; ${ }^{33}$ e como não-neutralidade perante o outro, ou seja como Alteridade. ${ }^{34}$

As respostas associadas à concordância associada ao mercado justo podem ser justificadas como sendo utilitaristas, ${ }^{35}$ desde que preservado o Princípio da Justiça. ${ }^{31}$

As quatro questões que referiram diferentes exemplos de mercado de órgãos atualmente existentes tiveram discordâncias como respostas predominantes. Essa discordância pode ser explicada pela questão da
Dignidade Humana. ${ }^{36}$ Não é aceitável transformar uma pessoa em apenas um meio para a obtenção de órgãos mediante benefícios financeiros diretos ou indiretos. A remuneração direta teve discordância maior que os benefícios indiretos.

Quando a questão mudou o foco da possibilidade para a eventual necessidade pessoal ou de um familiar, o referencial ético também se alterou, pois predominou a Ética das Emoções, ${ }^{29}$ em função do vínculo afetivo associado ao ato de obter órgãos para transplante. As categorias de respostas utilizadas permitem reconhecer que as pessoas que concordaram que pagariam em caso de necessidade se baseiam nessa perspectiva da Ética da Emoção; ${ }^{29}$ os que discordaram reiteram a posição já definida anteriormente de respeito à Dignidade Humana. ${ }^{36}$ Nessa questão foi verificada a maior frequência de pessoas que não tiveram opinião. Isto talvez possa ser explicado pelo Positivismo Moral, onde o que os outros pensam se sobrepõe a minha opinião pessoal, ou seja, assumir uma posição pode acarretar julgamentos. ${ }^{29}$

\section{CONCLUSÃO}

Com base nos resultados obtidos na presente amostra estudada, é possível concluir que há uma alta aceitação referente à doação solidária $(80,1 \%)$, corroborando com o atual sistema de doação de órgãos existente no Brasil e uma discordância com as diferentes abordagens de mercado apresentadas. Mas ao incluirmos o envolvimento de necessidade pessoal ou familiar para a obtenção de órgãos para fins de transplante, há uma aceitação significativa $(54,9 \%)$ ao pagamento para obtenção de órgão. Ou seja, coletivamente os participantes apoiam amplamente a doação solidária, condenam a possibilidade de pagamento ou remuneração associada à captação, mas a maioria das pessoas pagaria para obter um órgão em caso de extrema necessidade pessoal ou de seus familiares.

Esses resultados evidenciam a mudança de respostas em uma perspectiva pessoal ou coletiva, com múltiplas abordagens éticas de justificação. Isto demonstra necessidade de um aprofundamento da reflexão sobre os aspectos éticos associados às questões envolvidas na captação de órgãos para fins de transplantes. 


\section{ABSTRACT}

Purpose: This article presents the perceptions of health professionals and the public as to the method to obtain organs, especially the market approach. Methods: To preset data on the selflessly perception of the participants and including the involvement of personal or family need to obtain organs for transplantation by using the market approach. To meet the objectives of this study, a questionnaire was developed to collect the opinions of participants. Its distribution was personally performed in a random way, and it was also developed an electronic version released via Facebook page. The analysis of the responses were discussed at a $5 \%$ significance, and considered significant whenever $p$ was $<0.05$. Results: Overall, 692 people participated in the survey. In the health professional category there are more likely than among the general population to disagree on the indirect incentives related to the tax reduction and 30-days paid leave. These were the only significant associations related to health professionals. Most participants (80.1\%) agreed that organ donation must be a selfless act and strictly supportive, but $52 \%$ believe that the market could be a fair and beneficial system for all. In an extreme situation of absolute shortage of organs, $54.9 \%$ of respondents indicated they would pay for an organ to save his/her life or the life of a family member. Conclusion: Collectively, participants broadly support the solidary donation condemning the possibility of payment or remuneration associated to the funding, but most people would pay for an organ in extreme personal cases or upon a family need. This demonstrates the need for further reflection on the ethical problems associated to the issues involved in the organ procurement aiming to perform transplants.

Keywords: Gift Giving, Remuneration, Commerce, Transplantation

\section{REFERENCES}

1. WHO (2008). Declaração de Istambul sobre Tráfico de Órgãos e Turismo de Transplante. Istambul. Available from: http://www.declarationofistanbul.org/index.php.

2. Naím, M. Ilícito: o ataque da pirataria, da lavagem de dinheiro e do tráfico à economia global. Rio de Janeiro: Jorge Zahar; 2006: 150-1.

3. Lamb D. Transplante de Órgãos e Ética. Trad. Jorge Curbelo. São Paulo: Sociedade Brasileira de Vigilância de Medicamentos/Editora Hucitec, 2000.

4. Veatch RM. Transplantation Ethics. Washington DC: Georgetown University Press; 2002.

5. Goldim, JR. Aspectos Éticos dos Transplantes de Órgãos. 2005, Available from: http://www.bioetica.ufrgs.br/transprt. htm

6. Cardozo B. Dissenting opinion in Schloendorff v. Society of New York Hospital. 1914.

7. Góes PW. Transplante e comercialização de órgãos: limites à disponibilidade do corpo humano. I Simpósio de Análise Crítica do Direito, Universidade Estadual do Norte do Paraná, 2011.

8. Berlinguer G, Garrafa V. A mercadoria final: a comercialização de parte do corpo humano. 2nd ed. Brasília: Universidade de Brasília; 2001.

9. Brasil. Estado do Rio Grande do Sul. Lei Estadual 8.750, de 12 de dezembro de 1988.

10. Kerstein SJ. Are kidney markets morally permissible if vendors do not benefit? Am J Bioeth. 2014;14(10):29-30.
11. Ghods AJ. Governed financial incentives as an alternative to altruistic organ donation. Exp Clin Transplant. 2004;2(2):221-8

12. Novelli G, Rossi M, Poli L, Morabito V, Ferretti S, Bussotti $A$, et al. Is legalizing the organ market possible? Transplant Proc. 2007;39(6):1743-5.

13. Julian K. Assessing the Likely Harms to Kidney Vendors in Regulated Organ Markets. Am J Bioeth. 2014;14(10):718.

14. Alberto G. Harms to Vendors: We Should Discourage, Not Prohibit Organ Sales. Am J Bioeth. 2014;14(10):25-7.

15. Kiarash A. A Closer Look at the Iranian Model of Kidney Transplantation. Am J Bioeth. 2014;14(10):35-7.

16. Sigrid F. The Truth About Iran. Am J Bioeth. 2014;14(10):3738.

17. Atieh P, Farzaneh Z, Zeinab P, Bagher L. Paid Living Kidney Transplantation in Iran: Rethinking the Challenges. Am J Bioeth. 2014;14(10):40-42.

18. Alexander MC, Gabriel MS, Francis LD. Organ Markets: Problems Beyond Harms to Vendors. Am J Bioeth. 2014;14(10):23-25.

19. Erik M. A Further Lesson From Existing Kidney Markets. Am J Bioeth. 2014;14(10):27-29.

20. Samuel JK. Are Kidney Markets Morally Permissible If Vendors Do Not Benefit?. Am J Bioeth. 2014;14(10):29-30.

21. Monir M. Regulated Organ Market: Reality Versus Rhetoric. Am J Bioeth. 2014;14(10):33-35. 
Percepção da população em geral e dos profissionais de saúde sobre a forma de obtenção de órgãos para transplante: a perspectiva mercadológica

22. Julie A, Aviva G, Marie CF. Regulated Markets of Kidneys in Developed Countries or How to Increase Health Inequities. Am J Bioeth. 2014;14(10):44-45.

23. James T. Avoiding Harms to Kidney Vendors through Legal, Regulated Markets. Am J Bioeth. 2014;14(10):21-22.

24. Glenn C. A Fuller Picture of Organ Markets. Am J Bioeth. 2014;14(10):19-21

25. Benjamin H. All the More Reason: Why Julian Koplin Should Support a Trial of Incentives for Organ Donation. Am J Bioeth. 2014; 14(10):31-33.

26. Dominique M, Sarah W. Risk, Regulation, and Financial Incentives for Living Kidney Donation. Am J Bioeth. 2014; 14(10):46-48.

27. Farnan MJ. Connectivity and Consent: Does Posting Imply Participation?. Am J Bioeth. 2014;14(10):62-63.

28. US Department of Health and Human Services. National Survey Organ Donation Attitudes and Behaviors. Washington (DC): HRSA; 2013.
29. Varga AC. Problemas de Bioética. São Leopoldo: Unisinos, 1982:1-16.

30. Aristóteles. Ética a Nicômacos. 2nd ed. Brasília: Universidade de Brasília; 1992.

31. Beauchamp TL, Childress JF. Principles of biomedical ethics. 4th ed. New York: Oxford; 1994.

32. Bandman EL, Bandman B. Bioethics and Human Rights: A Reader for Health Professionals. Lantham MD: Univ Pr of Amer; 1984.

33. Jonas $\mathrm{H}$. O principio responsabilidade: ensaio de uma ética para a civilização tecnológica. Rio de Janeiro: Contraponto; 2006.

34. Lévinas, E. Entre nous: essais sur le penser-à-l'autre. Paris: Grasser \& Fasquelle; 1991.

35. Singer, P. Practical Ethics. 2nd ed. New York: Cambridge; 1993.

36. Immanuel Kant. Fundamentação da Metafísica dos Costumes. Lisboa: 70; 2005. 